Cahiers de philosophie de l'université de

\title{
Confiteor. Le retour à soi dans les Confessions de saint Augustin
}

\section{Emmanuel Housset}

\section{(2) OpenEdition \\ 12 Journals}

Édition électronique

URL : https://journals.openedition.org/cpuc/532

DOI : $10.4000 /$ cpuc.532

ISSN : 2677-6529

Éditeur

Presses universitaires de Caen

\section{Édition imprimée}

Date de publication : 31 décembre 2015

Pagination : 39-68

ISBN : 978-2-84133-746-0

ISSN : $1282-6545$

\section{Référence électronique}

Emmanuel Housset, "Confiteor. Le retour à soi dans les Confessions de saint Augustin », Cahiers de philosophie de l'université de Caen [En ligne], 52 | 2015, mis en ligne le 13 juin 2018, consulté le 28

février 2023. URL : http://journals.openedition.org/cpuc/532 ; DOI : https://doi.org/10.4000/cpuc.532

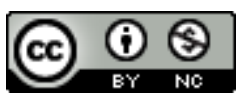

Creative Commons - Attribution - Pas d'Utilisation Commerciale 4.0 International - CC BY-NC 4.0 https://creativecommons.org/licenses/by-nc/4.0/ 


\section{Confiteor. Le retour à soi dans les Confessions de saint Augustin}

$\mathrm{L}$ E DÉSIR DE SE DIRE, de se raconter, de se faire objet de spectacle est un très puissant principe de mouvement, qui conduit à se comprendre comme le créateur de son être, dans un rêve de toute-puissance portant sur le monde et sur soi-même. Pourtant, il est possible de se demander si une telle idée de création de soi correspond vraiment à l'essence de la parole. La parole n'est-elle que ce pouvoir de représentation et d'expression de soi dont dispose l'homme, et ce pouvoir de parler ne vient-il que de soi? La parole n'est-elle que cet instrument dont se sert la personne pour maîtriser sa propre existence? Pour le dire autrement, la parole humaine, dans son essence, est-elle créatrice ou confessante? Cette alternative n'est pas une simple opposition historique entre une conception moderne et une conception médiévale de la parole, car elle porte en elle une interrogation sur l'essence de la parole comme sur l'essence du soi. Quel est le lieu de la parole? Quel est, pour reprendre une expression de Heidegger dans Acheminement vers la parole, le "parler pur» auquel on accède par une réduction de toutes les fonctions mondaines de la parole? Tout philosophe est en chemin vers ce qui lui donne la parole, vers l'être; cependant dans ce chemin quelle est la place de la parole sur soi, sur son moi de fait? Cette parole est-elle portée par un rêve de transparence sur soi, est-elle au contraire la reconnaissance d'une vie pulsionnelle insondable me privant de ma liberté, ou est-il possible d'envisager une troisième compréhension, qui déplace totalement la question en envisageant une figure très différente de l'ipséité dans laquelle il est mis fin à la solitude ontologique du soi? La question n'est pas simplement de savoir si j'ai accès ou non à moi-même, mais si en revenant à moi je ne trouve que moi dans ce chez soi.

Il arrive parfois qu'un philosophe raconte sa vie, mais quel est alors le sens de cette parole? Doit-elle être comprise comme un dérapage, comme un décrochage, comme une manifestation de la finitude humaine, qui fait qu'il est difficile de se tenir en permanence à la hauteur de l'universel? Si, 
comme cela est décrit par Husserl dans le second volume de la Philosophie première, l'entrée en philosophie est semblable à une conversion, car elle demande un renversement de son être tout entier à partir d'une décision ${ }^{1}$, cette conversion semble exiger que le philosophe mette entre parenthèses toute sa vie personnelle, pour devenir Selbstlos, littéralement "sans soi", en tant que spectateur désintéressé du monde et de lui-même. Si Husserl termine ses Méditations cartésiennes par une référence à saint Augustin afin de formuler l'impératif de revenir en soi pour découvrir la vérité, il n'en demeure pas moins que non seulement il donne une signification très différente à la proposition de saint Augustin, mais qu'en outre jamais ce retour en soi n'a pour lui la signification d'une attention à son moi de fait, puisqu'il s'agit de prendre conscience et de vouloir les Idées-fins absolues. Selon cette philosophie de la réflexion, il appartient à l'eidos du philosophe de ne pas parler de son moi empirique, car la fonction de l'universel est précisément de libérer de la particularité et de la factualité. Le moi empirique ne possède qu'une valeur relative, comme toutes les choses du monde, et l'homme, dont le philosophe est la norme, n'accomplit son essence qu'en visant les valeurs absolues. Dans cette perspective d'une métaphysique de la subjectivité, qui veut remonter à un ego transcendantal unique source de tout sens, parler de soi, pour un philosophe, ce serait retomber dans l'attitude naturelle et dans ce bavardage qui consiste à recouvrir le monde et les autres par le récit indéfini des péripéties de sa vie quotidienne. Pour une philosophie de la subjectivité pure et de la volonté pure, qui pose le principe que seules les questions gnoséologiques sont véritablement déterminantes, parler de son moi de fait ne peut avoir que le statut d'une chute, de l'incapacité à tenir l'exigence radicale de la réduction transcendantale, et de cette chute qui est toujours une rechute il est impossible d'en être définitivement protégé, car les préjugés fondamentaux de l'attitude naturelle peuvent être suspendus, mais ne peuvent pas être extirpés de la conscience. Cette compréhension du devenir soi, en tant que travail indéfini de purification de la conscience, ne peut comprendre l'attention au moi empirique que comme une perte de liberté, comme une véritable prison qui interdit tout accès à l'être absolu.

1. «Or ce radicalisme absolu, pour qui veut devenir philosophe en ce sens le plus authentique du mot, implique que lui incombe une décision correspondante qui engagera sa vie de façon absolument radicale, une décision qui fera de sa vie une vie par vocation absolue (Leben aus absoluter Berufung)» (E. Husserl, Philosophie première, $2^{\mathrm{e}}$ partie, in Husserliana, t. VIII, La Haye, Nijhoff, 1959, p. 11, trad. A.L. Kelkel, Paris, PUF, 1972, p. 15). Sur cette question de la vocation comme don de sa vie à l'Idée dans la philosophie de Husserl, qu'on me pardonne de renvoyer à mon ouvrage La vocation de la personne, Paris, PUF, 2007, chapitre XI. 
On peut voir dans cette considération la reprise d'un thème platonicien: le philosophe n'est pas un sage, il n'enseigne pas la vertu, et, en conséquence, il doit se retenir de présenter sa vie particulière comme un modèle, et cela ne serait-ce que parce que le mimétisme ne saurait être un principe de moralité. En effet, l'idée semble s'imposer d'une certaine obliquité de la recherche de la vérité, dans la mesure où le retour sur soi du philosophe le conduit à parler d'autre chose que de lui-même. Il est alors légitime de se demander si la biographie est vraiment un chemin pour le philosophe ou si elle n'est pas simplement un souci de sauver son passé de l'oubli et de se l'approprier dans une représentation. Quel peut être le sens philosophique du projet de se raconter soi-même? N'est-ce pas une entreprise totalement vaine? La philosophie, notamment de Pascal à Kant, n'est-elle pas ce qui montre, contre l'opinion, que ce récit de soi n'est qu'un mode inauthentique et extérieur du rapport à soi, qui construit la fiction d'une identité substantielle? On ne peut que constater combien une telle parole est le plus souvent instrumentale et n'a d'autre but que de construire une image de soi efficace dans le monde et qui bloque tout accès à son être. Tel est le paradoxe dont une réflexion sur le moi empirique des philosophes doit partir: parler de soi, se raconter, peut produire une forme de dépersonnalisation par la construction d'une image qui, dans un même mouvement, masque sa véritable singularité et interdit tout processus d'universalisation. Singularité et universalité seraient perdues en même temps dans la production de cette image de soi.

Plus encore, parler de soi, pour le philosophe, n'est-ce pas tomber dans cette vaine curiosité qui éloigne de la vérité? Pour reprendre une distinction célèbre du livre $\mathrm{X}$ des Confessions de saint Augustin, n'aimons-nous pas la vérité quand elle éclaire notre moi comme une chose pour assurer une gloire et une maîtrise et ne haïssons-nous pas la vérité quand elle donne à voir ce qu'il y a d'ombre en nous ${ }^{2}$ ? Les particularités de sa vie ne font-elles pas partie de ces choses dont non seulement ce n'est pas un péché de les ignorer ou de les oublier, mais dont on peut penser en outre que c'est un péché de vouloir les étudier ou de vouloir s'en souvenir? Sans évoquer ici la complaisance possible dans le récit de ses méfaits passés, le simple projet de vouloir se raconter n'est-il pas déjà un mal, une expression de l'orgueil ${ }^{3}$ ? En effet, on peut soupçonner que vouloir faire la lumière sur son moi empirique ne soit qu'une manière particulièrement vicieuse de

2. Rémi Brague commente souvent dans son œuvre cette distinction fondamentale; voir son livre Modérément moderne, Paris, Flammarion, 2014, p. 221-223.

3. Montaigne lui-même disait disposer d'une mauvaise mémoire. Voir A. Compagnon, Un été avec Montaigne, Paris, Éditions des Équateurs, 2013, p. 141-144. 
se cacher à la lumière qui m’accuse dans mon péché. Le lieu de la parole philosophique se trouve dans la manifestation même de la vérité et la question est de savoir si cela interdit toute parole sur son moi empirique ou bien si cela ouvre une autre parole sur sa singularité. Autrement dit, est-il envisageable qu'une parole de philosophe sur son moi de fait relève d'autre chose que de ce que saint Augustin nomme dans le livre X des Confessions la concupiscence des yeux, qui est la figure inversée de l'amour de la vérité, puisqu'il s'agit d'une avidité de savoir, d'une idolâtrie de la connaissance de soi? Saint Augustin met en lumière que le projet de tout savoir sur soi conduit à se transformer soi-même en objet de spectacle, à devenir pour soi-même un "voyeur» dans un manque de pudeur envers soi qui s'oppose au véritable souci de soi. Or cette vaine curiosité qui conduit à s'enfermer en soi, ou plutôt dans ce qu'on imagine être soi, est sans doute plus grave que l'erreur, car l'homme perd dans ce regard sur lui-même jusqu'à la possibilité d'un dévoilement de son être. Ainsi chaque personne est ce dont elle se préoccupe, et le philosophe qui vit vraiment dans l'amour de la vérité, s'il parle de lui-même, doit au préalable trouver le lieu d'où une telle parole ne brise pas sa vocation. Le plus difficile, ce qu'expérimente le philosophe, est peut-être de parler de soi sans se perdre. Comment parler de soi sans parler de soi, pour que cette parole, dans l'humilité, puisse être un lieu de manifestation de la vérité? Autrement dit, comment revenir à soi sans que ce retour se transforme, d'une façon ou d'une autre, en un culte de soi?

La lecture des Confessions de saint Augustin est alors un lieu privilégié pour affronter les questions qui viennent d'être évoquées, et pour être à l'écoute de ce texte si commenté il convient de satisfaire au moins à deux exigences. La première est certes de laisser à saint Augustin sa propre parole en évitant toute forme d'anachronisme et la seconde, liée à la première, est d'être conscient du présent à partir duquel nous interrogeons les Confessions. Dans sa formulation même, la question du moi empirique des philosophes est une question qui se pose sur un horizon kantien et à partir de la conceptualité mise en place par la Critique de la raison pure et qui permet d'assurer la perspective transcendantale ${ }^{4}$. Ainsi la distinction de la matière et de la forme dans leur signification transcendantale conduit à celle de l'empirique et du pur et donc à cette séparation du moi empirique et du

4. «J'appelle transcendantale toute connaissance qui s'occupe en général non pas tant d'objets que de notre mode de connaissance des objets en tant qu'il est possible en général» ( «Introduction» à Critique de la raison pure, trad. A. Delamarre et F. Marty, in Euvres philosophiques I, Paris, Gallimard (Pléiade), 1980, p. 777). 
je transcendantal'5. Il s'agit donc bien d'interroger le sens même d'une telle séparation: le philosophe n'est-il qu'un sujet théorique pur animé par un processus d'idéalisation et ainsi par l'exigence d'un dépassement de toute finitude, ou bien peut-il y avoir une signification positive du fini dans la mesure où le philosophe, sans être un idolâtre de son cher moi, continue à tenir une parole humaine sur la vérité? On voit bien ici qu'il en va avec cette question de l'essence de la parole: l'homme, dont le philosophe est la figure idéale, est-il la seule source de sa parole dans un travail indéfini d'universalisation, ou bien le philosophe est-il aussi celui qui répond à l'appel de la vérité à travers sa facticité et sa finitude? Celui qui lit aujourd'hui les Confessions de saint Augustin peut revenir sur lui-même et adresser à sa propre conception implicite du sujet, comme animé par une volonté pure d'idéalisation, la question suivante: n'y a-t-il pas une dignité du fini, notamment quand le philosophe témoigne de l'insertion temporelle de la vérité? Le philosophe ne peut-il pas être libéré de cette abstraction qu'est l'idée d'un sujet pur de toute finitude, dans la mesure où c'est toujours dans le temps qu'il s'agit de dire l'intemporel? Si comme l'a montré Kant jamais la considération du moi empirique ne pourra permettre de dire qui est la personne et quelle est sa dignité, est-il possible de développer une compréhension non anthropologique de la finitude et de la facticité?

\section{L'unicité des Confessions}

La très grande majorité des spécialistes ${ }^{6}$ de la pensée de saint Augustin s'accorde à dire qu'il y a un caractère tout à fait unique des Confessions, dans la mesure où cet ouvrage est à la fois extrêmement personnel dans les livres I à IX et pourtant n'a d'autre but que de parler de la présence de Dieu dans la vie humaine. On trouve dans les Confessions un accord tout à fait exceptionnel entre l'extrême singularité du récit et sa signification universelle qui fait que ce livre est inclassable.

Cela ne signifie pas qu'il n'y ait pas d'antécédents aux Confessions et le genre biographique consistant à décrire des vies de philosophes, dont

5. Le je pur de Kant et de Husserl est, comme pur centre de fonction, un sujet dépourvu de toute qualité, et en cela il n'est que la condition de possibilité de l'objectivité.

6. «C'est le caractère unique des Confessions, à la fois très subjectif, très personnel, et, en même temps, entièrement centré sur Dieu, qui explique la portée universelle de cette œuvre. Par leur tour subjectif, les Confessions touchent chaque homme dans son intimité personnelle et lui parlent de ses propres expériences, s'il a, du moins, assez de lucidité sur lui-même et de courage pour suivre la voie d'intériorité que décrit saint Augustin» (S. Pinckaers, En promenade avec saint Augustin: à la découverte de Dieu dans les Confessions, Les Planssur-Bex, Parole et Silence, 2002, p. 53). 
la méthode est de croiser la vie personnelle et la doctrine, était déjà bien développé7. De plus, le souci du philosophe de construire une image de lui-même, qui n'est jamais seulement physique, pour indiquer à partir de sa singularité l'humanité elle-même, traverse l'Antiquité tardive ${ }^{8}$. Il reste à savoir, et c'est une question qui est très discutée, si les Confessions entrent véritablement dans le genre autobiographique, ou si, comme de nombreuses grandes œuvres philosophiques, elles ne possèdent pas un style unique 9 . De fait, saint Augustin aura un biographe en Possidonius de Calame, cependant on peut défendre contre une certaine interprétation que les Confessions ne sont pas une biographie et encore moins un journal intime. Dans une thèse célèbre, Georg Misch, en 1950, dans l'édition augmentée de Geschichte der Autobiographie, soutient que saint Augustin donne naissance au genre autobiographique qui s'accomplira au XVIII ${ }^{e}$ siècle. Les récentes études augustiniennes ont montré qu'une telle thèse est difficile à soutenir, cela dit Georg Misch lui-même souligne la complexité des Confessions, car cette œuvre n'est pas une simple juxtaposition d'un récit personnel et d'une doctrine philosophique et théologique. Il ne s'agit sans doute pas non plus de proposer une illustration dramatique d'une question morale: saint Augustin ne se met pas en scène pour rendre visible une vérité morale. Enfin, comme le montre également Georg Misch ${ }^{10}$, les Confessions n'offrent pas une auto-interprétation d'un sujet se constituant lui-même, mais marquent une tentative pour se dire à partir de Dieu, sans se prendre pour autant pour Dieu. Hans Urs von Balthasar, quant à lui, insiste dans son introduction à l'édition allemande des Confessions ${ }^{11}$ sur le fait que la biographie est ici entièrement au service de la théologie. Autrement dit, si on réserve le terme d'autobiographie pour le récit d'un sujet sur lui-même, qui met en évidence les propres fins poursuivies par le sujet, les Confessions ne sont pas une autobiographie, mais une biographie dans laquelle la vie empirique de saint Augustin est considérée en vue des fins de Dieu. Il s'agit de mettre en évidence le "pour Dieu» de sa vie personnelle et donc sa signification théologique première. Hors de cette dimension théologique $\mathrm{du}$ sens, le projet des Confessions ne peut pas se comprendre.

7. Voir ibid., p. 46-47. Voir également l'ouvrage de Martial Gueroult, Histoire de l'histoire de la philosophie, I. En Occident, des origines jusqu'à Condillac, Paris, Aubier, 1983, p. 55-63.

8. Voir ici le beau livre d'Anca Vasiliu, Images de soi dans l'Antiquité tardive, Paris, Vrin, 2012, notamment p. 161.

9. Comme dit Proust dans Le temps retrouvé, le style est une question non de technique, mais de vision.

10. Geschichte der Autobiographie, Francfort-sur-le-Main, Verlag G. Schulte-Bulmke, 1950, erster Band, zweite Hälfte, p. 656-657.

11. Augustinus, Die Bekenntnisse, Stuttgart, Johannes Verlag, 1985. 
Où étais-je, moi, quand je te cherchais (ubi ego eram, quando te quaerebam)? Toi, tu étais devant moi; mais moi, j'étais parti loin de moi, et ne trouvais plus moi-même, moins encore, oh combien! toi-même ${ }^{12}$.

De ce point de vue, la totalité de l'ouvrage relève, toujours selon Hans Urs von Balthasar, d'une théologie dialogale, ce qui ne s'oppose pas du tout à son caractère spéculatif. Ainsi, toute la lecture de sa vie empirique s'effectue selon un complet renversement de perspective de l'homme sur lui-même, puisqu'ici il n'est pas lui-même la source de la lumière sur luimême, mais il tente de se comprendre à partir de la lumière de la foi ${ }^{13}$. Ce n'est certes pas une autre histoire que la sienne qu'il s'agit de raconter, mais en se retournant sur sa condition de créature il s'agit de prendre conscience de la façon dont Dieu agit en soi et est la source de sa liberté ${ }^{14}$.

Mais, de même qu'elle n'avait pas mérité devant toi d'être ainsi une vie qui pût être illuminée, de même, quand elle était déjà, elle ne mérita pas non plus devant toi d'être illuminée. Car l'informité de cette vie ne t'aurait pas agréé, si elle n'était devenue lumière, non pas en existant mais en contemplant la lumière illuminante et en adhérant à elle. En sorte qu'elle ne doit qu'à ta grâce, et simplement de vivre, et de vivre dans la béatitude, convertie par une mutation meilleure vers ce qui ne peut se muer ni en mieux ni en pis $^{15}$.

12. V, II, 2, p. 465. Les Confessions seront citées selon la traduction de la Bibliothèque augustinienne, t. 13 et 14 (Études augustiniennes, 1992).

13. S. Pinckaers, En promenade avec saint Augustin..., p. 71: «Les Confessions exposent la lecture que saint Augustin a faite des événements de sa vie sous la lumière de la foi et de l'expérience de l'action divine, une lecture qui suppose, en partie, un retournement des perspectives ordinaires d'un homme sur ce qu'il a vécu. Il lit sa vie non plus selon ce qu'il a fait lui-même, mais selon ce que Dieu a fait de lui, avec lui, maintes fois à son insu et malgré lui, et il s'aperçoit finalement que Dieu était derrière lui, agissant en lui ». Cela fait signe vers le fides facit personam de Luther : l'homme ne se définit pas par ce qu'il fait de lui, par ses actes, et la personne est d'abord l'œuvre de Dieu. Voir M. Vial, «Fides facit personam. La notion de personne chez Luther et quelques-uns de ses lecteurs contemporains", Les Cahiers Philosophiques de Strasbourg, 31, 2012, p. 107-132.

14. Heidegger lui-même « dé-théologise» les Confessions en ramenant ce texte à une autobiographie dialogale et en comprenant la confession comme une interprétation: "L' 'interprétation" comme interprétation de soi-même, caractérisée d'une manière déterminée : ce devant quoi l'on confesse ce qui se rapporte à "soi-même", n'est pas seulement le "devant quoi vide", mais dirige l'interprétation proprement dite, en en faisant justement une interprétation spécifique» (Phénoménologie de la vie religieuse, GA 60, p. 247, trad. J. Greisch, Paris, Gallimard, 2012, p. 279). Voir C. Sommer, «(Qui) Suis-je? Quaestio augustinienne et Seinsfrage Heideggérienne », in Le jeune Heidegger (1909-1926), S.-J. Arrien et S. Camilleri (éd.), Paris, Vrin, 2011, p. 171-184. Mais pour saint Augustin, ce n'est pas le soi qui se place devant Dieu; Dieu seul a l'initiative et me pose la question «Qui es-tu? ». Devenir soi, c'est s'incorporer cette question qui vient de Dieu et qui rend possible une interrogation sur ma facticité.

15. XIII, III, 4, p. 431. 
Les Confessions sont donc une biographie dans laquelle l'homme n'est pas la mesure de lui-même et dans laquelle il ne s'agit pas de construire son image, ni même d'avoir le projet de tout justifier. Or, force est de constater qu'il existe toute une littérature psychologisante qui s'étonne que saint Augustin ne dise rien de sa femme, pas même son nom, alors qu'il y a de nombreux passages sur sa mère Monique. Ces remarques passent totalement à côté du projet des Confessions, qui n'est en rien celui d'une maîtrise de soi par la totalisation continue de son passé, comme si l'homme était sa propre œuvre et pouvait se rendre transparent à lui-même. L'étrangeté et l'unicité des Confessions, notamment pour ceux qui voient dans la biographie la manifestation de la capacité humaine à se donner une identité stable, substantielle, au-delà des discontinuités de l'existence, tiennent au souci qui s'y affirme de devenir attentif à la manifestation d'une transcendance qui vient briser toute totalité construite, et c'est pour cela que des pans entiers de la vie de saint Augustin n'entrent pas dans ce récit et que seuls certains fragments constituent son histoire sacrée tout en participant à l'histoire du salut. Quel que soit l'attachement qu'il ait pu avoir pour sa femme, elle ne participe pas à sa conversion, alors que sa mère y joue un rôle décisif ${ }^{16}$. Cette question montre qu'il ne s'agit pas du tout ici de construire par réflexion l'histoire de sa vie, mais de tenter de repérer dans sa vie la façon dont le logos de Dieu s'incarne dans l'histoire: Dieu se révèle dans certaines dimensions de mon histoire personnelle, et c'est pourquoi je ne dois pas confondre en moi l'histoire profane et l'histoire sacrée dans laquelle Dieu est le sujet de l'histoire. La confession consiste bien à découvrir Dieu dans sa propre histoire de façon à pouvoir accomplir la mission unique qui est la sienne, ce qui veut dire être soi. Pour reprendre une distinction de MerleauPonty dans La Prose du monde, l'autobiographie serait une historicité de mort, car elle fige la personne en objet de musée, alors que la confession est une historicité de vie, qui reconduit l'homme à son origine. En cela, le récit des Confessions ne relève pas non plus de ce que Ricœur nomme une identité narrative, dans la mesure où la rencontre de Dieu remet en cause toute forme d'identité stable issue de l'homme lui-même, toute idée d'un rassemblement de sa vie dans l'unité du récit. Ce n'est pas seulement

16. «De fait, près de neuf ans se sont écoulés ensuite. Moi, je les ai passé dans cette boue des bas-fonds, dans les ténèbres de l'erreur, et, malgré de fréquents efforts pour me lever qui me laissaient plus lourdement, je m'y suis roulé; pendant ce temps cette veuve chaste, pieuse et sobre, comme tu les aimes, déjà plus allègre sans doute dans l'espérance, mais non moins assidue aux larmes et aux gémissements, ne cessait, à toutes les heures de sa prière, de se lamenter sur moi auprès de toi. Ses prières entraient en ta présence, et pourtant tu me laissais encore me rouler et m’enrouler dans ces ténèbres» (III, XI, 20, p. 403). 
la mêmeté qui se trouve mise en cause, mais également la possibilité de l'ipséité, si on réduit celle-ci à un face-à-face avec soi ${ }^{17}$.

La traduction même du titre est un enjeu philosophique. Une récente traduction propose de traduire par "Les aveux» ${ }^{18}$; Goulven Madec propose, lui, de traduire Confessiones par "Louanges ${ }^{19}$. Cette divergence confirme un point souligné par de très nombreux interprètes de la pensée de saint Augustin, à savoir que l'unité de l'ouvrage ne peut se donner à comprendre qu'à partir de cet acte de parole qu'est la confession, et cela vaut bien sûr également pour la troisième partie du texte, qui explique ce que l'Écriture fait connaître à l'homme sur Dieu et son œuvre ${ }^{20}$.

Jean-Louis Chrétien, en plaçant le verbe «confesser» au centre de son livre, met en lumière que la confession n'est pas un acte de parole parmi d'autres, mais est d'abord celui qui ouvre la possibilité des autres actes de parole $^{21}$. Là encore, de nombreuses publications ont montré l'apport propre de saint Augustin dans l'usage du terme "confession», dont le sens primitif était celui d'aveux, mais qui dans la langue chrétienne signifie de façon indissociable la profession de foi ${ }^{22}$, la reconnaissance de la condition de pécheur et l'action de louange ${ }^{23}$. Selon saint Augustin, l'aveu des péchés ne prend sens qu'à partir de l'acte de rendre gloire, de louer, comme l'indique le tout début des Confessions:

Te louer, voilà ce que veut un homme, parcelle quelconque de ta création, et un homme qui partout porte sur lui sa mortalité, partout porte sur lui le

17. Cela pourrait expliquer pourquoi les Confessions, comme œuvre, sont très peu citées dans l'analyse de l'identité narrative, et pourquoi Ricœur, comme bien d'autres, se concentre sur les livres X et XI en dissociant l'étude de l'essence du temps de la considération de la parole confessante. Le récit peut-il aider à surmonter la distension du temps du péché? Voir I. Bochet, Augustin dans la pensée de Ricœur, Paris, Éditions Facultés jésuites de Paris, 2004.

18. F. Boyer, Les aveux, Paris, POL, 2008.

19. G. Madec, Le Dieu d'Augustin, Paris, Cerf, 1998, p. 85. Ce débat autour de la traduction par «aveux» ou «louanges» est cependant loin d'être récent.

20. Voir sur ce point l'introduction et les notes d'A. Solignac dans l'édition de la Bibliothèque augustinienne.

21. J.-L. Chrétien, Saint Augustin et les actes de parole, Paris, PUF, 2002, p. 121: «La confession, avant d'être une parole de foi qu'on pourrait distinguer d'autres et comparer avec d'autres, forme l'ouverture même de la dimension où les paroles de foi deviennent possibles. Qu'elle soit cri, chant, demande, question, réponse, toute parole chrétienne (et plus généralement biblique) ne sera ce qu'elle est que pour être d'abord, et enfin, confessante».

22. XI, II, 2, p. 273: «Il y a longtemps que je brûle de méditer sur ta loi, et de t'en confesser ce que je sais et ce que j'ignore, ce que tu as commencé d'illuminer et ce qui me reste de ténèbres, jusqu'à ce que la force engloutisse la faiblesse».

23. Voir J. Ratzinger, "Originalité et tradition dans le concept augustinien de "confessio" ", texte de 1957 repris dans Saint Augustin, M. Caron (dir.), Paris, Cerf (Les Cahiers d'histoire de la philosophie), 2009, p. 9-36; P. Courcelle, Recherches sur les Confessions de saint Augustin, Paris, E. de Boccard, 1950; article "Confessio, confiteri », in Augustinus Lexikon, vol. 1, C. Mayer (éd.), Bâle, Verlag Schwabe, 1994, col. 1122-1134. 
témoignage de son péché, et le témoignage que tu résistes aux superbes. Et pourtant, te louer, voilà ce que veut un homme, parcelle quelconque de ta création. C'est toi qui le pousses à prendre plaisir à te louer parce que tu nous as fait orientés vers toi et que notre cœur est sans repos tant qu'il ne repose pas en toi $^{24}$.

La confession est une «reconnaissance» ${ }^{25}$, au double sens d'une gratitude et d'une exploration, ainsi qu'une miséricorde envers soi-même rendue possible par la patience de Dieu, et c'est pourquoi elle s'éloigne du sens païen de l'aveu. Bien évidemment, le christianisme n'invente pas la dimension humaine de l'aveu, mais il renverse le rapport à l'aveu, puisque la louange modifie le lieu même de l'aveu en faisant remonter la créature depuis sa facticité jusqu'au Créateur d'où elle provient.

Mais pourtant laisse-moi parler devant ta miséricorde; moi qui suis terre et cendre, laisse-moi pourtant parler, car voici que je m'adresse à ta miséricorde et non pas à l'homme qui rirait de moi, c'est à elle que je parle. Et toi aussi, peut-être, tu ris de moi; mais tourne-toi vers moi et tu auras pitié ${ }^{26}$.

Jean-Luc Marion, dans Au lieu de soi, dit en ce sens que les Confessions sont « une louange devenue texte » et cela dans un "texte à-Dieu ${ }^{27}$, qui n'a pas du tout le sens d'une narration selon Ricœur. Il y a là une conversion, puisque la parole humaine se trouve retournée dans et par la parole de Dieu $^{28}$. Le plan même des Confessions (livres I à IX: rappeler le passé de la naissance à la conversion; livre X: la mémoire du présent; livres XI à XIII : confession de l'origine et de l'avenir jusqu'au repos éternel en Dieu) ne peut vraiment apparaître qu'à celui qui comprend le nouveau rapport à la vérité:

Je veux «faire la vérité», dans mon cœur, devant toi, par la confession, mais aussi dans mon livre, devant de nombreux témoins (volo eam facere in corde meo coram te in confessione, in stilo autem meo coram multis testibus) ${ }^{29}$.

Dieu est non seulement l'objet des Confessions, mais il en est d'abord le sujet.

24. I, I, 1, p. 273.

25. Sur ce double sens de «reconnaissance», voir J.-L. Chrétien, Reconnaissances philosophiques, Paris, Cerf, 2010. Voir également M. Giusto, «La miséricorde envers soi-même: Siracide 30, 24(23) dans l'œuvre d'Augustin ", Revue d'études augustiniennes et patristiques, 59, 2013, p. 247-273.

26. I, VI, 7, p. 285.

27. J.-L. Marion, Au lieu de soi, Paris, PUF, 2008, p. 39.

28. Ibid., p. 58: «La confession revient ainsi toujours à se retourner vers Dieu indirectement d'abord en portant sur soi le regard même de Dieu». Bien évidemment, je ne peux pas me placer du point de vue de Dieu et me voir comme Dieu me voit, mais je peux apprendre à me regarder sous la lumière de Dieu.

29. X, I, 1, p. 141. 


\section{Un rapport décentré à soi}

Le renversement propre aux Confessions tient à cette thèse que le pouvoir d'être soi vient d'au-delà de soi, y compris dans la mémoire ${ }^{30}$. En effet, la conscience n'est pas dans la pensée de saint Augustin le lieu d'un dédoublement de soi, d'une Ichspaltung, mais elle est le lieu où Dieu parle et me parle ${ }^{31}$. La proximité à soi ne se laisse pas décrire ici comme un «je me pense» et l'élucidation du rapport à soi dans la philosophie de saint Augustin ne nous est accessible aujourd'hui que par une suspension de notre compréhension purement subjective et réflexive de la présence à soi. En effet, lire les Confessions demande un travail de mise entre parenthèses de la conception habituelle, pour notre époque «moderne», de la mémoire comme lieu d'une totalisation possible de soi par un travail de réflexion, car pour saint Augustin la mémoire, parce qu'elle est bien plus qu'un simple souvenir du passé et bien autre chose qu'un pouvoir de réflexivité, est en elle-même un lieu de conversion, de sacrifice à Dieu, et c'est pourquoi elle donne lieu à deux styles de vie: soit la distension du péché d'une mémoire gouvernée par l'orgueil, soit la temporalité du salut dans laquelle c'est l'avenir qu'est le Christ qui détermine le sens du regard vers le passé ${ }^{32}$. Saint Augustin offre ici une toute nouvelle compréhension du rapport à soi, dont l'expression culmine dans le texte le plus célèbre des Confessions:

Malheur! Malheur! Par quels degrés ai-je été entraîné aux profondeurs de l'enfer, oui d'un enfer de souffrance et de fièvre, faute de vérité, alors que c'est toi, mon Dieu - je te le confesse à toi qui as eu pitié de moi, même quand je ne te confessais pas encore - alors que c'est toi que, non pas en suivant les lumières de l'intelligence qui me met selon ta volonté au-dessus des bêtes, mais en suivant le sens de la chair, c'est toi que je cherchais! Mais toi tu étais plus

30. «Mais quoi de plus proche de moi que moi-même? Et voilà que la puissance de ma mémoire échappe à ma prise, alors que je ne puis exprimer ce moi lui-même sans elle» (X, XVI, 25, p. 185). Qu'il me soit permis de renvoyer sur cette question à mes deux chapitres sur saint Augustin dans L'intériorité d'exil, Paris, Cerf (La nuit surveillée), 2008.

31. Voir l'article "Conscientia» de Cornelius Mayer dans l'Augustinus Lexikon, col. 1218-1228. De ce point de vue, il est possible d'émettre quelques réserves sur certains aspects de la thèse de Jérôme Lagouanère d'une émergence du sujet dans l'œuvre d'Augustin, notamment «d'un sujet pensé comme réflexivité en acte et texte à déchiffrer» (Intériorité et réflexivité dans la pensée de saint Augustin, Paris, Institut d'études augustiniennes, 2012, p. 594). Toute la richesse spéculative des analyses augustiniennes sur la présence de l'âme à elle-même ne risque-t-elle pas d'être perdue dans une comparaison avec un concept de réflexivité qui appartient en propre à la modernité?

32. Une telle mémoire est alors compatible avec un oubli du passé (XI, XXIX, 39) et déplace le partage convenu du propre et de l'impropre: «Dieu par l'intermédiaire de qui nous apprenons que ce que nous croyons parfois nôtre nous est étranger, et que ce que nous croyons parfois nous être étranger est nôtre» (Soliloques I, 3, trad. S. Dupuy-Trudelle, in Euvres I, Paris, Gallimard (Pléiade), 1998, p. 191). 
intime que l'intime de moi-même, et plus élevé que les cimes de moi-même ( $t$ a autem eras interior intimo meo et superior summo meo $)^{33}$.

La confession est un renversement $\mathrm{du}$ "Connais-toi toi-même», et parce qu'elle libère de la recherche d'une vision pleine et entière de l'essence de l'homme, elle redonne une place à la particularité dans l'essence de l'âme. Elle permet alors d'échapper à une alternative qui semblait indépassable: ou s'élever à la contemplation de l'essence de l'homme en oubliant sa propre histoire, ou s'enfermer dans la contemplation du passé, ce qui conduit à finir comme la femme de Loth figé en statue, car on s'obnubile de façon idolâtrique d'une vie passée qui n'est plus la sienne et qui ne l'a peut-être jamais été. De ce point de vue, saint Augustin rompt avec le néoplatonisme, qui tend à réduire l'histoire de l'individu à une chute dans le multiple. En effet, c'est à la condition de ne pas ramener l'histoire à un simple récit des faits qu'il est possible de la comprendre comme un lieu de conversion et donc de retour vers l'unité divine. Ainsi, non seulement saint Augustin aide à surmonter le mépris de l'histoire, mais en outre il permet de ne pas tomber dans l'idolâtrie de l'histoire profane.

Il est essentiel que dans ce regard vers son passé qu'est la confession des péchés l'homme ne se juge pas lui-même, et c'est pourquoi les Confessions proposent tout autre chose qu'une nouvelle sagesse du monde fondée sur la connaissance de soi. En effet, la détermination de ce qui est mien dans les Confessions est inimaginable pour une sagesse du monde qui ne peut penser qu'une appropriation, puisque c'est là où je me perds, là où je m'oublie, là où je me donne, que je suis le plus proprement moi-même. La confession est une conversion ${ }^{34}$ qui est une mort à un certain rapport à soi finalement destructeur, mais également une renaissance. Selon cette perspective, le récit des Confessions relate trois conversions: la lecture de l'Hortensius et l'adhésion à la secte manichéenne (373), la lecture des livres des Platoniciens (386), c'est-à-dire la conversion à la philosophie, et enfin la décision de recevoir le baptême. Il ne s'agit pas d'une simple succession, ni d'une suite de réorientations, car les deux premières conversions préparent la troisième, et cela montre que c'est d'un état d'inquiétude, de déstabilisation, que peut avoir lieu le renversement des valeurs qu'est la conversion religieuse, qui reconnaît la primauté absolue de la Grâce. Or, cette grâce fait

33. III, VI, 11, p. 383 .

34. Au sens d'une metanoia et pas seulement d'une épistrophé. Comme le souligne Karl Barth, la confession n'est pas une simple pénitence, mais une existence sous le pardon de Dieu, car «seul le péché pardonné peut être véritablement connu et reconnu». Il n’y a pas de connaissance de soi sans cette conversion (Éthique 1, trad. P. Secrétan, Paris, Cerf, 1998, p. 138). 
que la conversion n'est pas un simple accomplissement de ce qui était déjà en préparation, et doit se comprendre comme une rupture dans laquelle l'homme devient autre que ce qu'il imaginait pouvoir être ${ }^{35}$. Le baptême est alors le centre de l'histoire personnelle du chrétien, le lieu où le sens de cette histoire peut se révéler ${ }^{36}$. Ces trop brèves analyses visent seulement à indiquer que le «je» auquel on accède par la lecture des Confessions n'est pas le «je pur» abstrait des opérations théoriques, ni le «je pur» principe de synthèse de l'immanence, ni bien sûr un simple moi factice, mais que c'est un «je» qui se prononce à partir du don de l'Esprit, et qui dès lors reconnaît que ce qui est à soi, c'est d'abord son péché et que par soi seul on est incapable de réaliser quelque bien que ce soit ${ }^{37}$. Par rapport à ce «je» du sujet moderne, qui dans sa volonté de création de soi par soi s'avance les mains pleines de ce qu'il a produit, la confession donne à penser un «je» qui reconnaît que ses mains sont vides et qu'il n'est possible de devenir soi que là où l'on ne se prend pas pour soi, car le retour à soi est déjà une Grâce, un don gratuit.

Bien évidemment, la confession, si elle est une parole à Dieu, n'a pas pour but de dévoiler à Dieu ce qu'il ignorerait, et le retour à soi vise à comprendre le mal comme sien tout en rapportant le bien à Dieu. Dès lors, une question peut se poser: comment ce retour sur soi sous la forme d'un aveu des péchés n'est-il pas désespérant? Face à cette difficulté, saint Augustin rappelle que la confession est indissociablement louange et donc qu'elle n'est pas un simple repentir qui condamnerait au désespoir face à notre malignité et à notre faiblesse. En effet, en plusieurs lieux, saint Augustin développe l'idée que dans le retour sur soi l'homme devient d'abord une question, un abîme ${ }^{38}$, tout en ajoutant qu'au cœur de cet abîme il est possible de rencontrer l'espérance. Ainsi, à travers la facticité et la finitude de son existence (et non pas malgré elle), l'homme peut découvrir ce qui l'excède et ce qui l'appelle. Ainsi, le retour sur sa vie personnelle

35. Voir le discours sur le psaume 121: «Et comme la charité détruit ce que nous étions, afin que nous devenions ce que nous n'étions pas encore, voilà que l'amour nous fait subir une certaine mort» (Discours sur les Psaumes II, trad. sous la dir. de M. Raulx, Paris, Cerf, 2007, p. 941).

36. Sur le caractère christocentrique de l'histoire du salut, voir O. Cullmann, Christ et le temps [1946], Paris, Delachaux et Niestlé, 1957, qui a donné lieu à de vives discussions sur le moment où le Christ est vraiment compris comme le centre de l'histoire.

37. «Mon péché venait, en fait, de ce que ce n'était pas en lui, mais dans ses créatures, en moi et dans les autres, que je cherchais voluptés, sublimités, vérités; et je sombrais ainsi dans les douleurs, les confusions et les erreurs» (I, XX, 31, p. 329).

38. «J'étais devenu moi-même pour moi une immense question, et j'interrogeais mon âme: pourquoi était-elle triste, et pourquoi me troublait-elle si fort? Et elle ne savait rien me répondre» (IV, IV, 9, p. 423). 
n'est pas un acte de réappropriation et permet de comprendre que «je ne puis pas moi-même saisir tout ce que je suis » ${ }^{39}$ et que «l'esprit est donc trop étroit pour se posséder lui-même ${ }^{40}$. Il ne s'agit pas là d'un simple constat d'un sujet s'observant lui-même, mais de l'étonnement originaire, de l'expérience première qui ouvre la possibilité de la temporalité du salut. En conséquence, un tel retour sur soi libère de tout regard désespérant (et finalement encore égocentrique) sur la finitude de l'esprit humain, car il est la découverte d'une grandeur qui ne nous appartient pas et qui constitue pourtant la «très secrète unité » ${ }^{41}$ de notre être. Notre unité ne vient pas de nous, mais de l'Idipsum et du Christ, qui en participant à notre être nous fait participer à cette unité ${ }^{42}$. En effet, Dieu est l'Idipsum, c'est-à-dire un soi que l'homme ne sera jamais, mais auquel il peut aspirer par sa mémoire, son intelligence et sa volonté. Il nous est donc nécessaire de revenir à notre vie empirique, au mal en nous, pour découvrir le plus haut; sans un tel retour, il est impossible de briser le cercle de la seule considération de soi, de la seule inquiétude pour soi, pour s'ouvrir à un mouvement de transcendance qui élève vers le Bien. Saint Augustin propose donc un tout autre regard sur la facticité: elle n'est ni une fatalité ni un destin, elle n'est pas non plus une simple contingence à dépasser, mais elle est le lieu où je découvre en même temps ma misère et la grandeur de Dieu, ma dissemblance et la possibilité d'une ressemblance qui sauve du désespoir ${ }^{43}$.

Il est manifeste cependant qu'il peut aussi y avoir une perversité à vouloir se connaître dans son indigence et sa laideur : l'aveu du mal peut n'être luimême qu'un vice brillant et même une certaine complaisance dans le mal. Comment faire en sorte que le récit du mal ne soit pas un exhibitionnisme et une façon de prendre plaisir au mal? Dans les Confessions, le célèbre récit du vol des poires ${ }^{44}$ n'est pas l'histoire d'une péripétie malheureuse

39. X, VIII, 15 , p. 167.

40. Ibid.

41. I, XX, 31, p. 329 .

42. Voir Discours sur les Psaumes II, p. 931-932.

43. Le père Marie-Dominique Chenu présentait ainsi la nouveauté de saint Augustin : «Tandis que Denys et les siens engagent le cosmos dans la dialectique même qui aboutit à l'extase au-delà de l'intelligible, Augustin, chrétiennement infidèle à Plotin, inverse le processus de l'ascension vers Dieu, qu'il découvre, lui, dans les profondeurs intimes de l'esprit, délivré de la dispersion des créatures et de l'angoisse du devenir. La mens n'est pas le nous grec du Timée ni de Denys» (La théologie au douzième siècle, 3 éd., Paris, Vrin, 1976, p. 117-118).

44. «Eh bien! moi, j'ai voulu faire un vol, et je l'ai fait; aucun dénuement ne m'y poussait, sinon le fait que j'étais dépourvu et dégoûté de justice, et engraissé d'iniquité. Car j'ai volé ce dont j'avais une provision, et de bien meilleure qualité; et je voulais jouir, non pas de l'objet que je recherchais par le vol, mais du vol lui-même et du péché. Il y avait, à proximité de notre vigne, un poirier chargé de fruits que ni leur beauté ni leur goût ne rendaient alléchants. Pour secouer cet arbre et le piller, notre bande de jeunes garnements organisa 
de l'existence, mais une exposition de la découverte de la concupiscence, qui est le pouvoir de se détourner de sa fin comme aversion pour le Bien. Il en va de même de l'analyse de la triple concupiscence au livre $\mathrm{X}$ des Confessions et Heidegger souligne que c'est bien ici que saint Augustin s'éloigne définitivement de la conceptualité néoplatonicienne tout en développant une compréhension de la possession de soi qui sera «diluée» par Descartes ${ }^{45}$. L'épreuve même de la facticité et de la finitude est la condition nécessaire pour prendre conscience que la conversion est le renversement d'une aversion qui est d'abord la nôtre et donc pour que le moi devienne une question. Dès lors, la confession n'est pas une complaisance dans le mal, car elle est la possibilité de convertir la concupiscence, qui n'est pas toujours mauvaise ${ }^{46}$, en charité, c'est-à-dire en passant d'une existence où je crois être pour moi et par moi à une existence qui est dans l'attente de ce qui est au plus intime que l'intime de soi et plus élevé que le haut de moi-même, donc une existence où je suis par et pour Dieu.

Le retour à soi est la voie du salut s'il a lieu sous la conduite de Dieu et n'est pas une simple interprétation de soi:

Et, averti par ces livres de revenir à moi-même, j'entrai dans l'intimité de mon être sous ta conduite (inde admonitus redire ad memet ipsum intravi

une expédition en pleine nuit - car, selon une lamentable habitude, nous avions prolongé jusque-là notre jeu dans les carrefours - et nous avons emporté de là une énorme charge de fruits; ce n'était pas pour nous en régaler, mais seulement pour les jeter aux porcs; et même si nous en avions mangé quelques-uns, l'essentiel était pour nous le plaisir attendu d'un acte défendu. Voilà mon cœur, ô Dieu, voilà mon cœur que tu as pris en pitié au fond de son abîme. Qu'il te dise maintenant, mon cœur que voilà, ce qu'il y cherchait pour que je fusse gratuitement mauvais, et qu'il n'y eut pas d'autre mobile à ma malice que la malice même! Elle était horrible, et je l'ai aimée; j'ai aimé ma perte, j’ai aimé ma déchéance; ce n'est pas ce que je poursuivais dans ma déchéance, mais ma déchéance même que j'ai aimée, âme lamentable qui m'évadais de ta forteresse pour courir à la ruine, puisque je convoitais non pas une chose par infamie, mais l'infamie» (II, IV, 9, p. 345-347).

45. M. Heidegger, Phänomenologie des religieusen Lebens, GA 6o, Francfort-sur-le-Main, Klostermann, 1995, p. 298; trad. J. Greisch, p. 340. Voir aussi p. 254; trad. p. 288: « Se représenter la possession radicale de soi-même comme un solipsisme hyperréflexif, ou quelque chose de semblable, est une erreur totale. Le soi "est" celui de la facticité historique intégrale qui vit dans son monde, avec et en lui». Philippe Capelle souligne que Heidegger élabore son concept de facticité à partir de cette lecture de saint Augustin, tout en laissant délibérément de côté la question de la mémoire de Dieu, comme si on pouvait séparer cette autre compréhension de l'ipséité de la mémoire de Dieu! Il est en effet difficile de suivre cette façon d'éloigner saint Augustin de la théologie (Finitude et mystère, Paris, Cerf, 2005, p. 160-161). Sur l'évolution du concept de concupiscence dans l'œuvre de saint Augustin et notamment sur l'idée que saint Augustin intériorise peu à peu la concupiscence, voir T. Nisula, Augustine and the Functions of Concupiscence, Leyde - Boston, Brill, 2012, où l'auteur met en évidence que la concupiscence est l'occasion du péché, mais pas le péché.

46. Sur l'idée que pour saint Augustin une certaine concupiscence peut être bonne, voir l'article «Concupiscencia » de G. Bonner, Augustinus Lexikon, col. 1114. 
in intima mea duce te): je l'ai pu parce que tu t'es fait mon soutien. J'entrai et je vis avec l'œil de mon âme, quel qu'il fût, au-dessus de cet œil de mon âme, au-dessus de mon intelligence, la lumière immuable, non pas celle qui est ordinaire et visible à toute chair, ni une sorte de lumière du même genre qui serait plus grande et qui aurait, par exemple, beaucoup, beaucoup plus de splendeur dans son resplendissement et remplirait tout de sa grandeur. Non, ce n'est pas cela qu'elle était, mais autre chose, bien autre chose que toutes nos lumières 47 !

C'est par l'Idipsum que nous pouvons nous poser la question «Qui sommes-nous? » et, en retour, nous n'existons devant Dieu qu'en devenant une question, une question qui vient de Dieu lui-même. Vouloir poser cette question "Qui suis-je? » à partir de soi, c'est encore passer à côté de notre finitude et c'est tomber dans tous les dangers de l'orgueil. En effet, le refus de concevoir Dieu comme substance ou sujet est bien ce qui conduit saint Augustin à une autre compréhension de l'âme humaine dans laquelle elle n'est pas elle-même un sujet, un support de facultés, car elle est une image de la Trinité. Dès lors, c'est bien parce que l'homme est à l'image et à la ressemblance de Dieu que toute conception narrative de l'identité, dans laquelle un sujet s'attribue les accidents de son existence, se trouve radicalement remise en cause. La confession elle-même n'est pas un événement factuel de sa vie, mais elle est de façon essentielle l'âme elle-même, l'âme en acte, l'âme comme histoire, c'est-à-dire comme totalité en mouvement. Ainsi que le dit Alain de Libera, il s'agit d'un mode périchorétique de l'âme et non d'un modèle attributif ${ }^{48}$. La périchorèse ou la circumcession des Personnes trinitaires est la vie originaire à partir de laquelle celle de la trinité de l'âme humaine devient pensable. Comme on l'a vu, la mémoire, au sens élargi de saint Augustin, n'est pas une faculté d'un sujet-substrat, mais elle est l'esprit lui-même en acte: il est cette mémoire, il ne contient pas en lui des actes de mémoire. Cela confirme l'idée qu'il est impossible de trouver une mesure en soi et que c'est au moment même où l'on éprouve cette absence de mesure que l'âme peut se tourner vers une chose qui n'est pas en elle. L'homme n'est pas lui-même dans l'acte de se souvenir de lui-même ou de se souvenir du monde et il ne peut se trouver que dans la mémoire de la simplicité de Dieu. La vraie mémoire de soi, celle qui n'est pas idolâtrique, c'est-à-dire qui n'est pas une dispersion même dans la tentative de rassemblement, se déploie à partir de la mémoire de l'éternité, et c'est pourquoi elle n'est pas vaine, pourquoi elle n'est pas une perte dans la multiplicité du passé.

47. VII, X, 16, p. 615-617.

48. A. de Libera, Archéologie du sujet. I Naissance du sujet, Paris, Vrin, 2007, p. 266. 


\section{Le miroir des Écritures}

Les analyses précédentes ont montré que «faire la vérité» n’a pas du tout le sens d'un examen de conscience pour saint Augustin, dans la mesure où tourner le regard vers le mal en soi-même n'a d'autre but que de pouvoir se tourner vers le principe de toute visibilité qu'est Dieu. Or, cette idée nouvelle que l'accomplissement de la vie intérieure a lieu dans l'attente de Dieu ne se laisse comprendre que par la lecture de l'Écriture, et notamment des épîtres de saint Paul. En effet, il y a là une condition essentielle de cette compréhension de soi à partir de la vie facticielle, qui n'était pas présente dans le néoplatonisme et qui sera perdue dans la modernité, car à partir de l'Écriture le sens de ses misères peut apparaître sous un jour nouveau. Ainsi Dieu est bien le sujet des Confessions, puisque par Dieu je deviens moi-même une énigme et que par les Écritures il m'est possible de retrouver un équilibre en Dieu au-delà de toute dispersion dans le multiple et de tout projet animé par l'orgueil visant à me donner par moi-même une identité stable. Les Écritures sont donc un miroir, mais pas au sens où Baudelaire ${ }^{49}$ dit que la mer est un miroir ou que Proust dit dans Le temps retrouvé que son livre est un miroir pour s'y lire soi-même. En effet, les Écritures comme miroir montrent que l'être soi suppose une désappropriation préalable, car elles invitent à se détourner de soi pour aller vers Dieu ${ }^{50}$. Il ne s'agit donc justement pas d'interpréter le vrai souci de soi par l'acte de faire abstraction de soi, mais comme l'acte de se comprendre à partir de la parole de Dieu et à partir de son pardon.

Je veux rappeler à mon cœur les hideurs de son passé et les charnelles corruptions de mon âme; non pas que je les aime, mais afin que je t'aime, toi, mon Dieu. C'est par amour de ton amour que je le fais; je repasse mes voies d'iniquité dans l'amertume de mon ressouvenir afin que tu me deviennes doux, ô douceur qui ne trompe pas, ô douceur de bonheur et de sécurité, toi qui me rassembles de la dispersion, où sans fruit je me suis éparpillé, quand je me suis détourné de toi, l'Unique, pour me perdre dans le multiple ${ }^{51}$.

Tu voulais ainsi me faire rechercher la joie sans dégoût, et, là où j'aurais pu l'atteindre, ne me faire trouver rien d'autre que toi, Seigneur, que toi qui ériges

49. Les fleurs du mal XIV, «L’homme et la mer»: «Homme libre, toujours tu chériras la mer!/ La mer est ton miroir; tu contemples ton âme / Dans le déroulement infini de sa lame / Et ton esprit n'est pas un gouffre moins amer».

50. Voir J.-L. Chrétien, Sous le regard de la Bible, Paris, Bayard, 2008, notamment p. 22 : «Face à la Bible toute appropriation doit en même temps être une désappropriation». Voir également l'ouvrage d'I. Bochet, Le firmament de l'Écriture. L'herméneutique augustinienne, Paris, Institut d'études augustiniennes, 2004.

51. II, I, 1, p. 333. 
la douleur en enseignement, frappes pour guérir et nous tues pour que nous ne mourions pas loin de toi ${ }^{52}$.

Dès lors, prendre conscience de la laideur de son âme, c'est d'une certaine façon déjà accéder à une certaine beauté en acceptant de ne plus se faire le centre de tout ${ }^{53}$. Il faut alors reconnaître que le retour à soi n'est possible que parce que les Écritures interrogent l'homme et qu'elles font de lui une existence qui est une question; par elles, c'est Dieu qui me demande ce qu'il est pour moi.

Or, pour moi, rester attaché à Dieu est mon bien, parce que, si je ne demeure pas en lui, en moi non plus je ne le pourrai; mais lui, demeurant en soi, rend nouvelles toutes choses. Et tu es mon Seigneur, puisque tu n'as pas besoin de mes biens ${ }^{54}$.

En cela, dans les Confessions, la parole de Dieu ne fait pas que reconduire saint Augustin à sa propre énigme, car à la faveur de cette question qu'il existe, elle l'interpelle sur sa vocation et lui permet ainsi d'en approcher.

Lorsqu'en effet je suis mauvais, ce n'est rien d'autre de le confesser à toi que me déplaire à moi-même; et lorsque je suis bon, ce n'est rien d'autre de le confesser à toi que ne pas l'attribuer à moi-même, puisque c'est toi, Seigneur, qui bénis le juste, mais qui d'abord d'impie le rends juste ${ }^{55}$.

Cela confirme bien que l'acte de confession est le principe d'unité des Confessions, y compris dans les livres X à XIII : la vraie théologie demeure

52. II, II, 4, p. 337.

53. Discours sur les Psaumes II, p. 464: «Donc, ô mon âme, tu ne peux être belle, qu'en faisant l'aveu de ta laideur à celui qui est toujours beau, et qui, pour ces temps, ne l'a pas été pour toi». Voir également p. 461-462: "Vous vous êtes revêtu de confession et de beauté". Le Prophète place avant la beauté la confession, qui est la beauté dans la beauté même. [...] Mais où pourrai-je me contempler, me diras-tu? Il t'a donné pour miroir ses Saintes Ecritures; c'est là qu'il est dit: “Bienheureux ceux dont le cœur est pur, parce qu'ils verront Dieu". Cette parole même est un miroir, vois si tu es ce que dit cette Ecriture; et si tu ne l'es point gémis afin de le devenir. Le miroir te remettra devant les yeux ton propre visage; et comme il ne te flattera point, ne te flatte point toi-même. Sa pureté te montrera ce que tu es; et si tu te déplais à toi-même, travaille à n'être plus tel. Te déplaire dans ta laideur, c'est déjà plaire à celui qui est parfaitement beau ». Voir aussi Confessions XIII, II, 3, p. 429: «De même en effet que, pour un corps, être n'est pas la même chose qu'être beau, sans quoi il ne pourrait être difforme; de même aussi, pour un esprit créé, vivre n'est pas la même chose que vivre sagement, sans quoi il serait immuablement sage. Mais il est bon pour lui d'adhérer toujours à toi, de peur que la lumière qu'il a obtenue en se tournant vers toi, il ne la perde en se détournant de toi, et ne retombe dans une vie semblable au ténébreux abîme».

54. VII, XI, 17, p. 619.

55. X, II, 2, p. 143. 
toujours une confession, c'est-à-dire une parole devant Dieu, ou encore une parole dans laquelle l'homme peut rechercher sa forme véritable en étant confronté au Créateur.

Selon cette nouvelle intelligence de la vie intérieure, les Confessions peuvent opposer un retour à soi animé par l'orgueil et un retour à soi animé par l'humilité. Certes, la rencontre des livres des Platoniciens est une étape dans l'élévation de l'esprit, mais cela montre également l'orgueil d'une recherche de Dieu sans le Christ médiateur ${ }^{56}$. Ainsi l'orgueil et l'humilité forment deux styles de vie et cette opposition traverse toutes les Confessions. En effet, l'humilité conduit à reconnaitre que tout le bien en soi est reçu, et les livres I à IX sur la mémoire du passé sont une prise de conscience des Grâces dont saint Augustin a pu bénéficier. Cela confirme une nouvelle fois que Dieu est bien le sujet des Confessions, car l'histoire de la vie de saint Augustin est l'histoire de la Grâce dans sa vie ${ }^{57}$. Confesser, c'est donc à la fois penser et exister en découvrant ce qui nous fait être, ce qui travaille de l'intérieur en nous, car cette action de la Grâce nous transforme plus sûrement que toutes nos décisions sans lendemain. Les livres XI à XIII eux-mêmes montrent comment la Grâce, depuis toujours, traverse et anime toute histoire personnelle, puisque l'amour de Dieu est l'origine du temps.

Selon cette nouvelle perspective, les Écritures enseignent que le retour à soi ne consiste pas à développer un lien naturel de l'âme avec l'Intelligence et l'Un, mais qu'il est par la Création, la conversion à une personne. Il est ainsi possible d'envisager une réunification personnelle qui mette fin à la dispersion dans le multiple, mais qui ne soit pas une purification; contre tout projet de purification antique ou moderne, saint Augustin met en évidence que l'accomplissement de soi consiste à donner un sens nouveau à sa vie sensible. Les Confessions, en cela, veulent dégager toute la signification de la première épître aux Corinthiens $(4,7)$ : «Qu'as-tu que tu n'aies reçu?».

Je me mis à lire, et je découvris que tout ce que j'avais lu de vrai chez les Platoniciens, était dit ici sous la caution de ta grâce, afin que celui qui voit ne se glorifie pas comme s'il n'avait pas reçu, non seulement ce qu'il voit mais encore de voir, - qu'a-t-il en effet qu'il n'ait reçu? - et afin que, toi qui es toujours le même, il soit non seulement stimulé à te voir, mais encore guéri pour te posséder; et que celui qui de loin ne peut voir, marche cependant dans la voie par où il puisse venir et voir et posséder ${ }^{58}$.

56. Voir VII, XX, 26.

57. Voir les analyses de P.-M. Hombert, Gloria gratiae. Se glorifier en Dieu, principe et fin de la théologie augustinienne de la grâce, Paris, Institut d'études augustiniennes, 1996, p. 113-118.

58. VII, XXI, 27, p. 639. 
Une telle question n'implique aucune forme d'effacement de sa vie personnelle dans l'universel et, bien au contraire, c'est par un retour sur son existence facticielle qu'il est possible de s'élever à un universel qui ne soit pas abstrait. Il s'agit en effet de laisser cette vie personnelle être reformée par un autre ${ }^{59}$. Ainsi le don gratuit de la création conduit à penser que l'on n'accède à soi que dans un acte d'humilité et non dans cette affirmation de soi par soi qui conduit à la plus profonde déchéance. Justement l'humilité est ce qui permet de restaurer une unité que nous avons détruite par notre orgueil.

Dans ce don de toi, nous nous reposons : là, de toi nous jouissons; notre repos est notre lieu. L'amour nous y élève, et ton Esprit qui est bon exalte notre bassesse la retirant des portes de la mort. Dans la bonne volonté se trouve pour nous la paix. Un corps, en vertu de son poids, tend à son lieu propre. Le poids ne va pas forcément en bas mais au lieu propre. Le feu tend vers le haut, la pierre vers le bas: ils sont menés par leur poids, ils s'en vont à leur lieu. L'huile versée sous l'eau s'élève au-dessus de l'eau; l'eau versée sur l'huile s'enfonce au-dessous de l'huile: ils sont menés par leur poids, ils s'en vont à leur lieu. S'il n'est pas à sa place, un être est sans repos; qu'on le mette à sa place et il est en repos. Mon poids, c'est mon amour; c'est lui qui m'emporte où qu'il m'emporte. Le don de toi nous enflamme et nous emporte en haut ${ }^{60}$.

Ici encore, le retour à soi consiste à passer de l'illusion d'une connaissance de soi par soi à la conscience d'être dans l'ignorance de ce que l'on est, pour se laisser enseigner par les Écritures ce que l'on peut devenir. Les Écritures ne mettent donc pas notre facticité entre parenthèses, mais elles nous montrent au cœur de notre facticité ce qui la transcende et nous rend véritablement humain. La situation de l'homme est d'être ni totalement endormi ${ }^{61}$, ni totalement éveillé, et la confession empêche

59. Voir P.-M. Hombert, Gloria gratiae..., p. 428.

60. XIII, IX, 10, p. 439-441.

61. «Cependant, toi, ô médecin de mon être intime, dis-moi clairement quel peut être le fruit de ce que je fais là. En effet, les confessions de mes fautes passées, que tu as remises et couvertes pour me rendre heureux en toi, en transformant mon âme par la foi et par ton sacrement, ces confessions, quand on les lit et qu'on les entend, remuent le cœur: elles l'empêchent de s'endormir dans le désespoir et de dire: "je ne puis"; elles le tiennent au contraire éveillé dans l'amour de ta miséricorde et la douceur de ta grâce, car cette grâce fait la force de tout être faible qui par elle prend conscience de sa faiblesse. Les bons prennent plaisir à entendre parler des fautes commises dans le passé par ceux qui en sont désormais libérés; s'ils y prennent plaisir, ce n'est pas parce que ce sont des fautes, mais parce qu'elles furent et ne sont plus. Quel fruit donc, mon Seigneur à qui chaque jour se confesse ma conscience, plus assurée dans l'espoir de ta miséricorde que dans son innocence, quel fruit attendre, je te prie, si je confesse aussi aux hommes devant toi par cet écrit encore, ce que je suis, non plus ce que je fus? De fait, le fruit de la confession du passé, je l'ai vu et je l'ai mentionné. Mais ce que je suis encore, à l'instant que voici, au moment précis de mes confessions, bien 
de s'endormir dans le désespoir d'une impuissance, car par le miroir des Écritures l'homme peut découvrir de quoi il est capable. Cette façon toute particulière de voir son visage à partir des Écritures donne également à comprendre le non-sens qu'il y a à parler de soi pour soi et le non-sens de la vaine gloire qui fait du moi une idole vide et inconsistante. L'amourpropre, l'acte de se plaire en soi, est ce mal qui habite en nous et qui est la racine de toutes les tentations; c'est lui qui peut faire du retour sur soi un enfermement en soi et proprement un enfer. Au contraire, par l'humilité, l'homme trouve sa tenue non en lui, mais dans l'acte même de confesser la vérité, parce que le Christ lui révèle sa vocation propre. Tel est tout le paradoxe des Confessions, qui est de mettre en lumière une parole sur soi, qui est particulièrement attentive à la vie empirique, et dans laquelle pourtant ce n'est pas de soi que l'on parle, ni à soi que l'on répond. Être à partir du Christ, comme nous y invitent les Écritures, est ce qui libère de tout vain projet autobiographique et en même temps de tous les phantasmes que nous prenons pour nous-même. En conséquence, l'humilité est vraiment le seul pouvoir qui appartient véritablement à l'homme et saint Augustin peut écrire: "Quoi donc? Serai-je en sûreté, quand je verrai ce que je ne pouvais voir? Serai-je parfait? Non, tant que durera cette vie. Notre perfection ici-bas, c'est l'humilité ${ }^{62}$.

\section{L'interpersonnalité des Confessions}

Au livre X, saint Augustin met en évidence le caractère communautaire de la confession: le «je» de la parole confessante est un «nous» et elle ne saurait être réduite à une parole privée sans se perdre, car ce serait encore une expression de l'orgueil.

Mais quel fruit cherchent-ils en voulant cela? Désirent-ils unir leurs actions de grâce aux miennes, quand ils apprendront combien je m'approche de toi par ta grâce, et prier pour moi quand ils apprendront combien je suis freiné par mon propre poids? Je me révélerai à des gens comme ceux-là. Oui, ce n'est pas un maigre fruit, Seigneur mon Dieu, qu'ils soient nombreux à te rendre grâce pour nous, et nombreux à te prier pour nous. Que l'âme fraternelle

des gens aussi désirent le connaître: ils me connaissent ou ne me connaissent pas, ils ont appris des choses de moi ou sur moi, mais leur oreille n'est pas contre mon cœur, là où je suis ce que je suis. Ils veulent donc apprendre par ma confession ce que je suis moi-même, au-dedans, où ils ne peuvent diriger ni l'œil, ni l'oreille, ni l'esprit; ils le veulent, prêts à me croire malgré tout, quant à me connaître?... et c'est la charité qui leur dit, en les rendant bons, que je ne mens pas dans mes confessions; c'est elle en eux qui me croit» (X, III, 4, p. 145-147).

62. Discours sur les Psaumes II, p. 1079. 
aime en moi ce que tu apprends à aimer, et qu'elle déplore en moi ce que tu apprends à déplorer. J'attends cette conduite de l'âme fraternelle, non pas de l'étrangère, non pas des fils d'une autre race dont la bouche a proféré la vanité et dont la droite est une droite d'iniquité, mais de l'âme fraternelle, celle qui en m'approuvant se réjouit sur moi et en me désapprouvant s'attriste pour moi; aussi bien, qu'elle m'approuve ou qu'elle me désapprouve, elle m'aime. Je me révélerai à des gens comme ceux-là. Qu'ils respirent à la vue de mon bien, qu'ils soupirent à la vue de mon mal. Mon bien, c'est toi qui l'as formé, toi qui me l'as donné; mon mal c'est moi qui l'ai commis, toi qui le juges. Qu'ils respirent à la vue du premier et qu'ils soupirent à la vue du second. Que des hymnes et des larmes montent à la fois en ta présence, venant des cœurs fraternels qui sont tes encensoirs. [...] Tel est le fruit de mes confessions, révélant non plus ce que je fus, mais ce que je suis; c'est que je le confesse non seulement devant toi, avec un secret transport de joie mêlé de tremblement, avec une secrète amertume mêlée d'espérance; mais encore aux oreilles des croyants, ces fils des hommes, associés à ma joie et participants de ma condition mortelle, mes concitoyens et les compagnons de mon voyage terrestre, qui me précèdent ou me suivent ou m'accompagnent dans la vie. Ce sont tes serviteurs, mes frères, et tu as voulu qu'ils soient tes fils, mes maîtres, que tu m'as ordonné de servir, si je veux avec toi vivre de toi. C'était trop peu pour moi que ton Verbe donnât cet ordre en paroles, s'il n'était aussi passé aux actes le premier. Et moi je l'accomplis en actes et en paroles; je l'accomplis sous tes ailes, car le péril serait bien trop grand, si je n'étais pas sous tes ailes où mon âme t'est soumise et ma faiblesse t'est connue. Je suis un petit enfant, mais mon père vit à jamais, et mon tuteur est qualifié pour moi; oui, c'est le même qui m'a engendré et qui m'a en tutelle, et c'est toi-même, toi qui es tous mes biens, toi le tout-puissant qui est avec moi avant même que je ne sois avec toi. Je révélerai donc à de tels hommes, semblables à ceux que tu m'ordonnes de servir, non pas ce que je fus, mais ce que je suis enfin et ce que je suis encore. Mais je ne me juge pas moi-même. Que l'on m'écoute donc dans cet esprit ${ }^{63}$.

Ce long texte qui précède les célèbres analyses sur la mémoire est en lui-même une élucidation du sens de la parole confessante: il s'agit de faire la vérité devant Dieu, mais également devant les lecteurs. Néanmoins la question se pose à nouveau: le récit de la facticité de sa vie personnelle n'est-il pas ce qui isole les hommes, ce qui les enferme dans leur singularité? N'est-ce pas l'universel et non la particularité qui est le fondement de la communauté, le lieu de l'être-ensemble? Encore une fois, en aucun cas saint Augustin ne cherche à faire de son histoire personnelle un modèle à imiter pour les autres hommes. S'agit-il même dans les Confessions de montrer

63. X, IV, 5-6, p. 147-151. 
l'Homme à partir de sa vie singulière et est-il possible de dire à partir de la construction d'une image de soi «ecce homo » sans retomber dans l'orgueil? En effet, les Confessions sont d'abord une admonitio, un appel à écouter en soi la vérité. Selon cette perspective propre à la philosophie chrétienne, la parole confessante n'est vraiment mienne que dans la mesure où je ne parle pas de moi, mais également parce qu'elle transmet la Parole de Dieu qui ne peut être entendue que dans la charité. Il ne s'agit donc pas d'une parole qui s'élargirait pour devenir intersubjective, car elle est en réalité d'emblée communautaire: elle n'est personnelle qu'en étant déjà interpersonnelle.

Voilà où j'en suis! Pleurez avec moi et pleurez pour moi, vous qui avez en vous-mêmes, au-dedans, quelque souci du bien, souci d'où sortent les actes; car si vous ne l'avez pas, tout ceci ne vous touche pas. Mais toi, Seigneur mon Dieu, entends, regarde, vois, aie pitié, guéris-moi, toi sous les yeux de qui je suis devenu pour moi-même un problème! Et voilà bien mon mal ${ }^{64}$ !

Contre les mirages de l'amour-propre, il faut bien reconnaitre que le récit de son histoire propre ne peut, à première vue, intéresser personne d'autre que soi, sinon les rieurs ${ }^{65}$ pour lesquels je suis un objet de spectacle. Ainsi, parler de soi, raconter ses malheurs ne fonde pas une communauté, mais est une parole qui n'a de sens que si elle est en elle-même déjà commune et saint Augustin renverse le sens de la facticité dans un «nous les faibles et les indigents»: dire sa souffrance devant Dieu et devant de nombreux témoins permet de s'en libérer non en la supprimant, ou en faisant comme si elle n'était rien pour moi, mais en retrouvant dans ce "nous» la possibilité de décider de son sens. En effet, parce que la parole n'est pas que ma parole, mais cette parole commune du "Nous les faibles et les indigents", elle est bien autre chose qu'un pouvoir de me désigner moi-même en construisant une image de moi, car elle est d'abord le lieu où, au cœur de ma facticité, la Vérité travaille en moi. Or c'est bien dans cette parole commune qu'il m'est possible de redonner sens à ma vie, un sens à mon histoire, en répondant avec les autres hommes à la Parole de Dieu. En cela non seulement la confession n'est pas la parole d'un homme isolé, mais en outre la confession appelle la confession comme une parole vraie appelle une parole vraie. C'est pourquoi les Confessions ne sont pas un récit que saint Augustin ferait pour lui-même et leur sens même est d'appeler à la

64. X, XXIII, 50, p. 233.

65. «Moi, cependant, je veux te confesser mes hontes, à ta louange! Permets-moi, je t'en supplie, et donne-moi de parcourir, dans mon souvenir présent, les parcours sinueux de mes erreurs passées, et de t'immoler une victime de jubilation. [...] Eh bien! Qu'ils rient de nous, les forts et les opulents! mais nous, les faibles et les indigents, nous voulons te faire confession» (IV, I, 1, p. 409). 
confession, à cette existence devant Dieu dans laquelle la concupiscence devient charité. Là encore, je ne peux pas me donner moi-même à moimême comme si j'étais le créateur de moi-même, et c'est pour cela que j'ai besoin de la parole confessante des autres, non pour identifier leurs histoires à la mienne, mais pour ne pas désespérer et pour comprendre que le salut est possible. Cependant, c'est toujours la médiation du Christ qui donne à comprendre que le prochain, qu'il écoute ma confession ou qu'il se confesse devant moi, est lui également un médiateur, qui me reconduit à moi-même en me reconduisant à Dieu.

Saint Augustin ne cesse de le rappeler tout au long des Confessions : le récit de sa vie n'a de sens que parce qu'il montre que dans sa vie lui aussi a tout reçu et que la conversion est elle-même un don gratuit de Dieu. Ainsi dans le partage de cette expérience les hommes s'édifient ensemble. Telle est alors la question qu'il convient toujours de se poser quand on en vient à raconter sa vie: est-ce pour sa propre gloire ou pour louer Dieu? Dans les Confessions, il est clair qu'il s'agit uniquement de donner accès au sens de la Création ${ }^{66}$. On comprend alors pourquoi cela ne pose pas de difficulté que la vie décrite par saint Augustin soit bien celle d'un indigent et d'un faible, et donc tout le contraire d'une vie illustre qui serait proposée comme modèle. Or une telle vie indigente est précisément ce qui n'enferme pas le lecteur dans un idéal de perfection à réaliser par un travail de purification et, au contraire, reconduit au Christ qui est le seul chemin. La description du moi empirique de saint Augustin, si on me permet cette expression anachronique, a donc une fonction d'exhortation, et si saint Augustin veut faire la vérité devant de nombreux témoins, ce n'est pas pour qu'on l'écoute lui, mais pour que nous puissions être à l'écoute de cette Parole dont il tente d'être à l'écoute.

Se peut-il aucunement, Seigneur, puisque l'éternité t'appartient, se peut-il que tu ignores ce que je dis, ou que tu voies selon le temps ce qui se passe dans le temps? A quoi bon alors ces récits détaillés que je te fais de tant d'événements? Ce n'est pas, bien sûr, pour que tu les apprennes de moi; mais j'excite pour toi mon amour et celui de ceux qui me lisent, pour que nous disions tous: Le Seigneur est grand, et tout à fait digne de louange! Je l'ai dit et le redirai : c'est par amour de ton amour que je fais ce récit. Car nous prions aussi, et pourtant la Vérité dit: Votre Père sait de quoi vous avez besoin avant que vous ne lui fassiez votre demande. Ce sont donc nos sentiments envers toi que nous dévoilons en te confessant nos misères et tes miséricordes sur nous, afin que tu achèves de nous délivrer, puisque tu as commencé; afin que nous cessions

66. Voir J.-M. Fontanier, Lectures des Confessions I-IV de saint Augustin, Rennes, Presses universitaires de Rennes, 1999. 
d'être malheureux en nous-mêmes et devenions heureux en toi, puisque tu nous as appelés; afin que nous soyons des pauvres en esprit, des doux, de ceux qui pleurent, qui ont faim et soif de la justice, des miséricordieux, des cœurs purs, des pacifiques ${ }^{67}$.

Ce principe détermine la méthode de «réduction» propre aux Confessions: tout ce qui ne reconduit pas à cette autre Parole est écarté du récit, est mis entre parenthèses, pour donner à entendre cette Parole qui nous donne la parole. C'est peut-être ici que l'on mesure l'abîme qui sépare un tel récit de sa vie finie de toute construction d'une identité narrative à partir du pouvoir unitaire d'un «je pur». Le «je pur» ne possède pas de pouvoir d'exhortation; s'il décrit la possibilité inconditionnée de prendre conscience de soi, il n'ouvre pas cette possibilité, et dans sa solitude il ne donne à penser l'acte de devenir soi que comme l'accomplissement indéfini d'une essence. Par rapport à cette perspective «moderne», le «je» de saint Augustin dans son dialogue avec Dieu n'est pas le «je pur» des opérations théoriques, mais un «je» qui invite à rentrer en soi, au cœur de sa finitude, pour trouver autre chose que soi, c'est-à-dire ce qui nous donne d'être.

\section{Une herméneutique de la finitude}

Les Confessions montrent donc que la vie de chaque jour est une vie effective, notamment dans toute sa dimension affective: c'est en elle que le sens de son existence se joue. Ainsi saint Augustin nous montre que le plus quotidien peut devenir le lieu de la vérité philosophique et théologique. Dans cette nouvelle conceptualité, l'incarnation n'est pas un mal, le temps n'est pas une simple dégradation de l'éternité, et l'existence dans sa particularité peut renvoyer, dans l'humilité, à l'être par un autre dans un rapport de personne à personne. Dès lors, si saint Augustin nous offre sa facticité, c'est pour que nous puissions devenir des pauvres capables de Dieu. On voit bien ici que la grande nouveauté des Confessions se trouve dans un tout autre rapport à la facticité: elle n'est pas à écarter, elle n'est pas non plus un sujet d'admiration, elle n'est pas indifférente, ni un objet de curiosité.

Il s'agit donc bien de penser une nouvelle forme du rapport à soi dans laquelle l'âme humaine n'est pas divine: le devenir soi ne consiste pas à se purifier pour faire resplendir la partie divine de son âme. L'homme cesse de se comprendre de façon grecque par sa place dans le cosmos et il se découvre lui-même en tant qu'intériorité qui accueille la vérité et qui aime. La longue patience du devenir soi consiste alors à transformer l'homme charnel en

67. XI, I, 1, p. 271. 
homme spirituel, en surmontant les trois concupiscences, ce qui suppose de se laisser éclairer par une vérité qui nous humilie, c'est-à-dire qui nous dévoile tel que nous ne voulons pas nous voir, à savoir faibles et indigents.

Il y a toujours en effet ces ténèbres déplorables, dans lesquelles se cache à mes yeux le pouvoir qui est en moi. C'est au point que mon esprit, s'interrogeant sur ses propres forces, n'ose pas trop se faire confiance à lui-même; car, ce qui réside en lui demeure le plus souvent dissimulé, si l'expérience ne le lui révèle, et par ailleurs personne ne doit être en sécurité durant cette vie qui est appelée d'un bout à l'autre une épreuve: celui qui de pire a pu devenir meilleur, ne pourrait-il aussi de meilleur devenir pire? Il n'y a qu'une espérance, une assurance, une promesse ferme: ta miséricorde ${ }^{68}$.

Selon cette perspective théologique, le Christ est la forme, mais aucun homme n'est le Christ, et, encore une fois, le Christ n'est pas du tout la figure idéale d'une perfection. Ainsi, les Confessions veulent donner à voir qu'à la difformité du péché peut répondre la conformation à l'image du Fils. L'homme est d'abord une image déformée et obscure, mais par l'écoute de la Parole il peut devenir une forme lumineuse et belle: "Cette nature, la plus noble parmi les choses créées, une fois purifiée de son impiété par son Créateur, quitte sa forme difforme pour devenir une forme belle ${ }^{69}$.

Toutes les Confessions montrent comment Dieu peut se montrer en sa forme, à savoir par le Fils, la «forme du serviteur», qui est la voie vers la vision finale de la forme de Dieu ${ }^{70}$. Il s'agit donc bien d'être libéré de soi, au sens d'être libéré de la forme que l'on se donne par soi, pour recevoir sa vraie forme en laissant parler sur soi une autre parole que la sienne:

Qu'y a-t-il donc entre moi et les hommes pour qu'ils entendent mes confessions, comme si eux devaient guérir toutes mes langueurs? Race curieuse de connaître la vie d'autrui, paresseuse à corriger la sienne! Pourquoi cherchentils à entendre de moi ce que je suis, eux qui ne veulent pas entendre de toi ce qu'ils sont? Et comment savent-ils, à m'entendre parler moi-même de moi-même, si je dis vrai, puisqu'aussi bien nul ne sait parmi les hommes ce qui se passe dans l'homme, si ce n'est l'esprit de l'homme qui est en lui? Mais s'ils t'entendent parler sur eux-mêmes, ils ne pourront dire: le Seigneur ment. Qu'est-ce en effet que t'entendre parler sur soi-même, sinon se connaître soi-même? Et qui donc, s'il connaît, peut dire «c'est faux», à moins qu'il ne mente lui-même? Mais parce que la charité croit tout, entre ceux-là du moins qu'à elle-même elle attache ensemble et rend «un», moi aussi, Seigneur,

68. X, XXXII, 48, p. 227-229.

69. La Trinité, XV, VIII, 14, p. 459.

70. Voir Augustinus Lexikon, article «Conformatio». 
encore une fois c'est ainsi que je te dis ma confession, pour qu'entendent les hommes à qui je ne puis prouver si, oui ou non, je dis vrai; mais ceux-là me croient qui ont pour moi des oreilles qu'ouvre la charité ${ }^{71}$.

Si l'homme doit comprendre qu'il est pour lui-même une grande question, ce n'est pas pour passer d'une image de soi à une autre image de soi: se comprendre, c'est se comprendre comme inimaginable, car créé à la ressemblance de Dieu qui est incompréhensible ${ }^{72}$.

Alors que Husserl décrit l'accès à soi par une altération fondamentale qui fait passer de la réflexion naturelle à la réflexion transcendantale, saint Augustin donne à penser une tout autre voie en montrant que le «je» du philosophe est inséparable de son moi empirique, et cela pour plusieurs raisons: d'une part, c'est tout entier, c'est-à-dire avec son histoire, sa faiblesse et sa finitude, que l'homme va vers sa vraie forme, et, d'autre part, c'est tous ensemble qu'un tel salut est possible. Néanmoins, si l'homme ne peut pas par lui-même adopter un point de vue absolu sur lui-même, s’il se découvre non dans l'infini de la réflexion pure, mais dans la liberté finie de son rapport au monde, si donc il n'est pas à lui-même sa propre lumière, c'est parce que son être n'est pas dans la possession, mais dans le don. Ainsi, ce n'est pas seulement l'épreuve de la tentation, l'expérience du poids de sa vie facticielle qui fait de moi une question, mais cette parole commune de la confession dans laquelle la question de l'être se pose à la première personne du pluriel. En effet, découvrir dans son être au monde ce qui excède le «je» par son immensité, à savoir l'événement du Christ qui donne sens à son histoire, cela revient bien à se comprendre comme faible et indigent, et seul celui qui dans sa facticité comprend qu'il a tout reçu peut se donner, en se libérant de tout projet d'abolition de sa finitude.

De lui, pèlerins d'ici-bas, nous avons reçu le gage pour que nous soyons déjà lumière, maintenant que sauvés, encore qu'en espérance, nous sommes devenus fils de la lumière et fils du jour, non plus fils de la nuit, ni des ténèbres, ce que pourtant nous avons été. Entre ceux-ci et nous, dans cet incertain où se trouve encore la connaissance humaine, toi seul fait le partage, toi qui éprouves nos cœurs et appelles la lumière jour et les ténèbres nuit. Qui en effet nous discerne, si ce n'est toi? Mais qu'avons-nous que nous n'ayons

71. X, III, 3, p. 145 .

72. Nous renvoyons bien sûr ici à la thèse constante de Jean-Luc Marion, et qu'il reprend particulièrement dans $A$ u lieu de soi (p. 146), en soulignant que l'homme ne ressemble pas à Dieu en ressemblant à quelque chose de visible ou d'intelligible. Il s'agit de porter la ressemblance du style de Dieu et non d'imiter une prétendue image de Dieu. Il y a, insiste Jean-Luc Marion, une indéfinition de l'homme qui appartient à son mode d'être. Voir également, du même auteur, Certitudes négatives, Paris, Grasset, 2010. 
reçu de toi, pris à la même masse et faits vases d'honneur alors que d'autres y sont pris en vases d'ignominie ${ }^{73}$ ?

La nouveauté et l'unicité des Confessions est peut-être là : le «je» des Confessions n'est pas un «je pur» réappropriable par toute subjectivité parce que hors d'être, mais un «je» inappropriable qui reconduit chacun à la tâche de se comprendre depuis sa finitude. C'est pourquoi elles ne donnent pas à voir de pures vérités théoriques, mais une vérité pratique qu'il s'agit d'exister à partir de la finitude de l'action. Le Christ ne donne Dieu à voir qu'en le donnant à être, et cela dessine un tout autre rapport à soi et à la vérité. Seule la reconnaissance de ce sens christique de la confession ouvre à une compréhension du chez soi comme habité par le visage de Dieu, et donc à une ipséité au-delà du principe d'identité. Le retour à soi n'est donc pas le retour à son "chez soi ", si on entend par là une sphère close dans laquelle je suis seul auprès de moi-même. La confession est en effet ce retour, à travers l'intelligence de sa vie empirique comme trace de l'invisible, au don premier et gratuit de mon être. En elle l'homme, au lieu de se complaire en lui-même, apprend à se déplaire, afin de perdre toute forme qui viendrait de lui et de recevoir sa vraie forme de la grâce de Dieu. Alors au moi creux ${ }^{74}$ construit par l'amour-propre se substitue une vie qui se remplit de l'amour des biens véritables venant de Dieu. L'homme en chemin ne cessera pas pour autant d'être pour lui-même une énigme, mais c'est le sens de cette énigme qui se modifie dans la mesure où il peut comprendre qu'il n'est vraiment lui-même qu'en laissant vivre l'amour de la vérité en lui. Ainsi l'homme n'est pas la raison de sa propre histoire et en conséquence son rapport à l'histoire est paradoxal: il se met à distance de son histoire profane et il acquiesce à son histoire sacrée, sans qu'il puisse $\mathrm{y}$ avoir une transparence de l'existence historique par une fusion de ces deux histoires ${ }^{75}$.

Comme l'écrit encore saint Augustin, la confession elle-même est un projet de Dieu qui veut guérir les blessures de l'homme et non les lui épargner. Dans cette guérison, le «je» se transfigure en un «me voici». Il faut plonger au plus profond du mal en soi, visiter tous les recoins de sa misère, car à partir de cette mort il est possible de renaître de la Parole pour tenter de se voir avec les yeux de Dieu. C'est pourquoi la confession ne prend

73. XIII, XIV, 15, p. 453.

74. X, XXXIX, 64 .

75. La réponse à la distension du temps n'est pas cette extension à laquelle nous fait encore rêver le projet réflexif d'identité narrative, mais c'est au contraire l'attente dans la patience qui fait que notre vie participe à l'histoire comme totalité. Voir H.U. von Balthasar, Das Ganze im Fragment, 2 éd., Fribourg-en-Brisgau, Johannes Verlag, 1990. 
son sens que dans la participation à la mort et à la résurrection du Christ. Ainsi la prière est ici l'acte fondateur de l'intériorité, comme le montre Jean-Louis Chrétien qui précise: «Par la prière la "chambre du cœur" devient le "temple de l'esprit" » ${ }^{76}$. En conséquence, le retour à soi n'est pas une perte de soi dans le culte de soi quand il prend la forme d'un sacrifice de sa particularité, d'un don de soi au prochain et à Dieu. L'humilité, et non plus l'orgueil, est alors bien le seul fondement du soi, et on ne revient à soi qu'en laissant Dieu agir en soi.

Emmanuel Housset

Université de Caen Normandie Identité et Subjectivité (EA 2129)

76. J.-L. Chrétien, L'espace intérieur, Paris, Minuit, 2014, p. 73. Il ajoute p. 128 : «Vivre activement l'espace intime qui est celui de notre esprit comme "temple", et en son centre comme un autel, c'est là une des plus hautes pensées de la dignité humaine, laquelle ne peut se renverser en culte de soi ». 